\title{
Una perspectiva feminista sobre las prácticas médicas frente al aborto en mujeres con tuberculosis y cáncer: Buenos Aires en las primeras décadas del siglo XX
}

\author{
A feminist perspective to medical practices. Abortion in women with tuberculosis \\ and cancer, Buenos Aires in the first decades of the twentieth century
}

\author{
María Laura Rodríguez \\ correodelaura80@hotmail.com \\ Centro de Investigaciones y Estudios sobre Cultura y \\ Sociedad (UNCyCONICET) - Universidad Nacional \\ de Córdoba, Argentina
}

Recepción: 12 Diciembre 2019

Aprobación: 08 Abril 2020

Publicación: 01 Septiembre 2021

Cita sugerida: Rodríguez, M. L. (2021). Una perspectiva feminista sobre las prácticas médicas frente al aborto en mujeres con tuberculosis y cáncer: Buenos Aires en las primeras décadas del siglo XX. Descentrada, 5(2), e149. https://doi.org/10.24215/25457284e149

\begin{abstract}
Resumen: Se recupera el contexto previo y posterior a la despenalización del aborto terapéutico en 1921, colocando en perspectiva las relaciones de saber y poder que constituyeron el cuerpo y el sujeto femenino. Si bien la legalización del aborto médico a partir de la reforma del Código Penal argentino fue una norma permisiva para su época, no habría ampliado las posibilidades de las mujeres a preservar su salud y vida sobre la gestación. Más aún,los profesionales médicos articularon concepciones y prácticas de clara tendencia conservadora, reconocibles tanto en una faz científica como político-ideológica.

Palabras clave: Aborto terapéutico, Género, Médicos, Tuberculosis y cáncer, Buenos Aires en las primeras décadas del siglo $\mathrm{XX}$.

Abstract: Recover the context of decriminalization of the rapeutic abortion in 1921 putting in to perspective the relationships of knowledge and power that constituted the body and the female subjects haped. Although legalization of medical abortion after there form of the Argentine Penal Code it was a permissive norm for its time, would not have extended the legal possibilities of woman to preserve their health and life over pregnancy. Furthermore, medical professionals articulated conceptions and practices with a clear conservative tendency, recognizable both on a scientific and political-ideological side.
\end{abstract}

Keywords: Therapeutic abortion, Gender, Doctors, Tuberculosis and cancer, Buenos Aires in the first decades of the twentieth century.

\section{INTRODUCCIÓN}

El artículo 86 del Código Penal de la Nación (CPN), que entró en vigencia en enero de 1922, determinó en qué circunstancias el aborto se encontraba despenalizado. A pesar de que esta normativa fue reconocida por su amplia permisividad para la época, la práctica del aborto legal ha resultado restrictiva y aun hoy continúa inaccesible para miles de mujeres, violando, en algunos casos, hasta el derecho a la vida (CELS, 
2016). De hecho, este tipo de tendencia se recordó recientemente al producirse el inadmisible veto a la resolución que protocolizaba la ILE en los últimos días del gobierno de Mauricio Macri en Argentina.

La introducción de la no punibilidad del aborto terapéutico habilitó un derrotero de investigación productivo desde la perspectiva histórica feminista. Esta búsqueda estuvo atenta a las tensiones de género, procurando explicar de qué maneras las ideas sobre la diferencia sexual se habían cargado de poder en la producción del conocimiento y en la práctica médica. Hace más de una década Diana Maffia (2006, p. 2) planteó que el inciso $1^{\circ}$ del artículo 86 del CPN de 1922, al establecer que el aborto no será punible "si se ha hecho con el fin de evitar un peligro para la salud o vida de la madre", encuadró una propiedad relacional, transformando a una mujer gestante, por ese sólo hecho, en "madre", dándole "a esta relación un fuerte carácter biológico y determinista en reemplazo de la función maternal". Como se sabe, la ley nunca es neutra en términos de género, e invariablemente, "las transgresiones ocurren por la omisión de garantías legales que padecen las derechohabientes" (Rosenberg, 2010, p. 6) Sin soslayar estos puntos, a lo largo de esa historia de sesgos y vacíos legales, resulta una cuestión de primer orden indagar las condiciones en las que los agentes de salud restringieron el acceso a la interrupción legal del embarazo. Sus concepciones y actitudes habrían sido particularmente determinantes en los años en que la indicación y la práctica eventual de la interrupción legal del embarazo estaba exclusivamente tutelada por los criterios de los profesionales intervinientes en el caso clínico (Cepeda, 2017, p. 2).

En este artículo se plantea que las persistentes reticencias que ha venido manifestando la corporación médica frente a la interrupción de los embarazos por razones de salud o de vida, respondieron a barreras socioculturales conformadas en un proceso histórico de largo plazo. Para demostrarlo se coloca en perspectiva un conjunto de artículos de revisión y de presentación de casos clínicos difundidos entre 1919 y durante toda la década de 1920 en la Revista Argentina de Obstetricia y Ginecología. Sus autores fueron distintos profesionales que trabajaban en el Hospital Rivadavia, en la Maternidad y en la Escuela de Parteras de La Plata, en el Hospital Rawson y en el Nacional de Clínicas. Se examina un conjunto de discursos y actitudes que condicionaron los criterios y las prácticas especializadas en el tratamiento de las embarazadas afectadas de tuberculosis y con cáncer de cuello de útero. Inicialmente, se tensionan supuestos desarrollados en trabajos previos (Rodríguez, 2018), que interpretaron las tendencias conservadoras de la gestación que predominaron en los casos de tísicas atendidas en algunos hospitales y maternidades de Buenos Aires, como una respuesta acorde a una matriz eugenésica positiva. A pesar del lugar central que se le dio a la llamada "eugenesia preventiva” como parte del programa social que imperó en la Argentina, como han planteado Marcela Nari (2004) y Graciela Quierolo (2013), tanto las campañas de "profilaxis social", como las concepciones y las intervenciones especializadas tuvieron visibles marcas de género. En respuesta a este panorama, se descubre que, más allá de ciertos discursos facilitadores sobre el aborto terapéutico, las posturas, las decisiones e intervenciones médicas frente a las enfermas gestantes estuvieron ancladas en criterios y prácticas altamente discrecionales, atravesados por persistentes sesgos de género.

\section{El ABORTO COMO Un ASUNTO MÉDico}

El artículo 86 estableció que la interrupción del embarazo por razones de salud y de vida era no punible, solo sí, era practicado por un profesional diplomado (Maffia, 2006). Según Sol Calandria y Nadia Ledesma Prietto (2018, p. 111) esta autoridad otorgada a los galenos para interrumpir embarazos, les confirió más poder como "únicos agentes legítimos" para intervenir sobre el cuerpo de la mujer. En este contexto, sostuvieron las autoras, "los representantes de la elite médica" apoyaron dicha legalización, puesto que, "el problema" para ellos se presentaba cuando el aborto remitía a "un acto voluntario" (p.111). Sin embargo, la conocida imbricación entre el proyecto profesional y el estatal, así como la particular aversión de los médicos con relación al llamado aborto "criminal", no alcanzaría a explicar en qué sentidos y con qué alcances ellos habrían sostenido la despenalización de los abortos no punibles. En el presente artículo, se 
aborda dicha complejidad partiendo de un conjunto de artículos médicos publicados en la Revista Argentina de Obstetricia y Ginecología que articularon nociones y actitudes extra científicas, atravesadas por una suerte de cautela legal que colocaba en segundo plano la salud y la vida de la mujer, potenciando la autonomía profesional sobre la norma legal referida.

En la primera década del siglo XX, el aborto terapéutico ya formaba parte de las preocupaciones de los médicos que atendían a embarazadas enfermas en reconocidos hospitales y maternidades de Buenos Aires. Si bien no fue posible dar cuenta de lo sucedido en cada uno de los nosocomios existentes, las evidencias muestran a distintos profesionales reconociendo la posibilidad de realizar abortos terapéuticos y, en algunos casos, practicándolos, desde bastante tiempo antes de la reforma del Código Penal de 1921 (Rodríguez, 2018). Puede resultar un poco más dificultoso revelar sus prácticas, sobre todo si la expectativa en ese sentido descansa en el ofrecimiento de un número cuantioso de historias clínicas. No cualquier intervención de este tipo habría tenido un interés científico suficiente como para convertirse en un tópico entre las publicaciones especializadas. Sin embargo, se han hallado ejemplos de este tipo de interrupciones del embarazo durante los años en estudio. Solo por mencionar algunos casos, que sean distintos a los repasados en el presente trabajo o en investigaciones propias anteriores (Rodríguez, 2018), se apuntan los identificados en indagaciones de Diego Armus (2007 y 2016a) y Yolanda Eraso (2007), aunque esta última autora señaló encontrar en ellos razones eugénicas antes que terapéuticas. Este último tipo de lecturas requieren algunas precisiones.

En ese orden, se recuperaron algunos aportes reconocidos por la historiografía sobre tuberculosis en Argentina. De acuerdo con Armus, el estudio del doctor Ricardo Schwarcz del año 1939, reveló que en la maternidad del Hospital Tornú durante el período 1925 a 1935, "los abortos artificiales de mujeres tuberculosas embarazadas aumentaron de modo sostenido" (2016a, p. 52). De acuerdo a ese escenario, este médico católico alegó que, en 1935, en el contexto de una nueva dirección en aquella institución, el aborto artificial fue formalmente descartado como práctica, sin que ese cambio haya producido aumentos en la mortalidad de la parturienta tuberculosa, tendencia que lo llevó a concluir "que el aborto artificial no parece haber traído ningún beneficio a la tuberculosa embarazada y sí, en cambio, haber servido durante unos pocos años para 'destruir la vida de muchos niños' (2016a, p.162). En ese sentido, Armus afirmó que "solo durante unos pocos años, el aborto de las tuberculosas parece haber tenido alguna presencia en las prácticas médicas institucionalizadas" (2016a, p.162).

Si bien este ejemplo de un profesional que trabajaba en el Hospital Tornú reforzó la lectura sobre la no excepcionalidad de las prácticas de abortos terapéuticos, también visibilizó una discusión relacionada con la interpretación que puede dársele a este tipo de intervenciones, en una época en la circularon "voces que impulsaban la idea de regular la sexualidad de los tuberculosos usando 'procedimientos negativos"' (Armus, 2016a, p. 162). Estos temas se trataron en un debate historiográfico que rehabilitó Armus (2016a), partiendo de recuperar los aportes que han venido subrayando la dominante presencia de la eugenesia positiva en la Argentina moderna. En tal sentido, el autor remarcó la necesidad de tensionar discursos y prácticas al analizar un conjunto de intervenciones médicas, como los abortos o esterilizaciones. En esa línea, una de sus criticas fue dirigida a la interpretación de Eraso, señalando que la autora, a pesar de haber contado con "pocas" historias clínicas que la respaldaran, asumió que en “(...) el hospital los médicos se las ingeniaron para practicar abortos terapéuticos con el argumento de prevenir la transmisión de caracteres hereditarios degradantes de la raza nacional" (2016, p.165). Aunque, tal como enfatizó Armus (2016a), no sería viable asumir que “(...) la existencia de un discurso debe entenderse, sin más, como la existencia de una práctica” (p. 165), algunas de sus lecturas deberían revisarse. A propósito de ello, en el presente artículo se insiste en que el valor historiográfico de la interpretación de Eraso no habría girado alrededor de defender la presencia dominante de la eugenesia negativa en la medicina argentina, sino más bien, en plantear la profunda discrecionalidad con la que se movían los profesionales al tratar a las mujeres enfermas. En efecto, este tipo de improntas colaboró en explicar las posturas adoptadas por los médicos ante el aborto terapéutico, sin involucrar la postulación de matrices eugénicas "negativas". 
Soslayando cuáles fueron las intenciones de cada uno de los profesionales, asunto prácticamente imposible de determinar, las fuentes consultadas dejaron ver que el aborto terapéutico constituyó un recurso habilitado por la ciencia médica, rastreable desde tiempos en que Hipócrates había aconsejado interrumpir el embarazo ante algunas situaciones mórbidas en que la gestante corría riego de salud o vida. En el número inaugural de la Revista Argentina de Obstetricia y Ginecología, el Dr. Armando Frers (1919), Director de la Maternidad y Escuela de Parteras de la Plata, dedicó un extenso artículo de revisión a caracterizar cómo el aborto médico había recorrido un largo camino hasta convertirse en un recurso social y jurídicamente legítimo en manos de la medicina.

Ahora bien, huelga decir que este propugnado interés social y legal excluía a la mujer como sujeto. Las concepciones de los especialistas no sólo habrían respondido a convicciones personales, más bien, daban cuenta de toda una agenda de modernización (y medicalización) eminentemente conservadora en términos de género (Giordano, 2013). Durante aquella época imperaban políticas sociales y de salud que afirmaban la moral católica y la ética médica, con un foco particular en la atención de la salud de madres e hijos. Pero, aquellos años y esas políticas también fueron cruciales para dar impulso a construcciones más sutiles y perdurables sobre el sujetofemenino, identificado crecientemente como madre. De manera particular, la ginecología y la obstetricia, legitimadas científica y políticamente, concibieron a las mujeres a partir de su aparato reproductivo y de su misión "natural" de perpetuar la especie (Nari, 2004).

Como han resaltado Carolina Biernat y Karina Ramaciotti (2013, p. 67) la salud de las mujeres, como "reales o potenciales" madres, devino en un objeto de preocupación e intervención por parte del Estado argentino desde las últimas décadas del siglo XIX. Fue en esa dirección, que "las políticas implementadas se organizaron en torno a un modelo promaternal" que, durante las décadas de 1920 y 1930, se orientó alrededor de una serie de ideas propias de la ideología poblacionista, tendientes a "proteger la familia y a desvincular a la mujer del mercado de trabajo", procurando el resguardo de los hijos a través de las madres (Biernat y Ramaciotti, 2013, p. 68). De acuerdo con estas premisas, se ha pensado en el aborto legal, reparando en este contexto de avance médico estatal por reforzar y garantizar la maternalización de la mujer. Julieta Arosteguy (2018) ha sostenido que la reforma del Código Penal de 1922, en lo que refiere al inciso que legalizó el aborto terapéutico, se encuadró en ese tipo de "previsión sanitarista de preservar la vida de la mujer en su doble rol de procreadora y cuidadora de la familia" (p.143).

Claro que, bajo el arbitrio exclusivo de los profesionales de la salud, aquel presupuesto de protección a la vida de la madre enferma fue una prerrogativa discrecional, dispuesta a partir de significativos sesgos de género. Durante la década de 1920 la conformidad que los profesionales dieron al aborto médico, si bien se afirmaba discursivamente en el objetivo de salvaguardar a la madre, soslayaba de plano cualquier asunto relativo a la decisión de la enferma gestante. Luego de 1922, los interrogatorios médicos narrados en las historias clínicas sugieren que la obligación legal que existía para que la paciente prestara consentimiento frente a la posibilidad de interponer el aborto era letra muerta. Los artículos científicos no ofrecen datos que indiquen si la mujer era informada - y cómo - de su riesgo de salud o vida. Antes, y también luego de la despenalización de 1922, el aborto terapéutico apareció como un asunto exclusivamente de interés médico que, como tal, reafirmaba un contexto en que la mujer no era considerada como una persona autónoma "con la capacidad moral y jurídica de tomar decisiones por sí misma” (Arosteguy, 2018, p. 144). Esta normativa funcionaba así, aun para resolver sobre temas de salud y vida.

Durante toda la etapa en estudio, las reconocidas "causales de salud o vida" fueron presentadas como un equivalente de "razones médicas", y la decisión de indicar y realizar un aborto interpelaba sólo los intereses de la elite galena como grupo corporativo. De hecho, ciertos médicos sostenían que este recurso podía ser ofrecido por la medicina gracias al exitoso desarrollo de una agenda de progreso. Para 1919, ese tipo de legitimidad se convirtió en un eje en la disputa por la secularización de la autoridad sobre la vida de las madres. Según la epopeya que se narró, la medicina había progresado desde un tiempo en que los médicos, por influencia de la Iglesia, habían preferido "dejar morir la parturienta antes de colocar el gancho en feto 
vivo", a otro, en que, "bajo el imperio de poder civil [se] había visto la completa aceptación de este recurso [e indignaba] la idea de que no se esté en la obligación de salvar la madre” (Frers, 1919, p. 430).

De acuerdo a lo explicó Frers (1919), el aborto terapéutico constituía un asunto estrictamente de incumbencia profesional. Incluso, algunos años antes de su legalización, este médico platense sostuvo que la ley penal argentina lo consentía

porque sabiéndolo, lo calla, y no lo ha tratado en especial como al aborto criminal, ni lo ha reglamentado, por la misma razón que no lo hace con las mutilaciones quirúrgicas en general, cuya falta de intención criminal lo coloca fuera de sus fronteras [art. 6 del Código Penal] (Frers, 1919, p. 431).

Pero, mientras el silencio estatal era concebido como una autorización implícita que respaldaba la separación entre las esferas civil y religiosa, este mismo vacío legal sustentaba algunas desconfianzas entre los mismos profesionales. La Sociedad de Obstetricia y Ginecología de Buenos Aires puso en discusión en varias oportunidades un temario de resquemores, alertas y propuestas de protección para médicos. Esta agenda no dividió las aguas entre ginecólogos y obstetras. Años antes de la sanción de la reforma penal de 1922, fue el Dr. Josué Beruti (1919), titular de la Cátedra de Clínica Obstétrica de la Facultad de Medicina de la Universidad de Buenos Aires y por entonces también Director de la Revista Argentina de Obstetricia y Ginecología, quien insistió en la necesidad de concentrarse en la parte estrictamente profesional de la cuestión. Su objetivo era "que el médico operador pueda actuar en tales circunstancias con una mayor tranquilidad moral y una mayor seguridad y garantía de los actos que realiza en tan delicadísimo asunto" (1919, p. 480-481). Frente a la posibilidad de indicar y practicar un aborto terapéutico se debatieron distintos mecanismos. Según recordaron los médicos presentes en la Sesión de la Sociedad de Ginecología y Obstetricia de octubre de 1919, desde hacía tiempo se venían considerando distintas vías de solución, como la organización de concilios de cinco o seis médicos para decidir la práctica a seguir y la hospitalización obligatoria de las pacientes en situación expectante. Muchos de estos puntos habían sido descartados porque se consideraron "impracticables". En cambio, fueron discutidas otras medidas, tales como la posibilidad de intervención de una autoridad oficial sanitaria, la implementación de la denuncia obligatoria donde debían constar los fines terapéuticos perseguidos con la intervención y la implementación de un mecanismo de interconsulta con otro facultativo. Se buscó en este último caso que la responsabilidad eventual de indicar y practicar un aborto, “estuviera más equilibradamente repartida entre el médico operador y otro especialista” (Beruti, 1919, p. 482).

En los años inmediatamente anteriores a su despenalización, las discusiones también se enfocaron en despejar al aborto terapéutico de cualquier alcance eugenésico (Beruti, 1919, p. 480-81). Ahora bien, aquel panorama de desconfianza no quedaría fácilmente zanjado en 1922. Evidentemente, el avance estatal comprometía la autonomía de la que venía gozando la élite médica. Como ha mostrado la historiografía, varios años después de reforma del CPN, algunos profesionales manifestaban que la normativización del aborto a través de la ley, había confundido asuntos de patología y clínica con los problemas legales, posibilitando que el ejercicio médico pudiera ser considerado un acto criminal (Rojas, 1930 citado en Rodríguez, 2009). Además, la novedad legal no pautó protocolos de actuación profesional.

Alrededor de este tipo de asuntos se actualizaron las discusiones de ginecólogos y obstetras, bajo una agenda que se declaraba interesada en "profesionalizar" la decisión y la práctica del aborto terapéutico. En los años finales de la década del 1920, surgieron acuerdos entre los profesionales que buscaron establecer marcos más generales para sus prácticas. Según el profesor de Clínica Obstetricia de la Facultad de Medicina de la Universidad Nacional de Buenos Aires, Juan B. González, en 1928 un congreso médico realizado de Chile discutió y aprobó un Código de Moral Médica estableciendo como condición preferente, aunque no excluyente en casos urgentes, la participación de dos facultativos para la decisión terapéutica de realizar abortos en casos de enfermas gestantes. (Rodríguez, 2018). Para 1934, cuando recién se consideró la introducción del aborto terapéutico en la propuesta para reformar la Ley que venía rigiendo en materia 
de ejercicio de la medicina y artes del curar en la provincia de Córdoba, se propuso que era necesario apoyar la "conciencia del médico actuante" en medios objetivos, como radiografías, fotografías y exámenes de laboratorio (González, 1934, p.178). La incorporación del aborto no punible en la discusión de esta legislación médica señaló de modo concluyente que los médicos constituyeron un factor central en la interposición de barreras para la concreción de esta práctica, en contexto de reciente cambio del estatus legal del aborto.

\section{CRITERIOS CIENTÍFICOS Y RACIONALIDAD PATRIARCAL}

En términos de la ciencia médica, el aborto no era aconsejable de igual forma en todas las condiciones patológicas que pusieran en riesgo la salud y la vida de la embarazada enferma. En el listado de enfermedades que habilitarían la posibilidad de aborto terapéutico, se distinguían las enfermedades consideradas de origen ovular y aquellas otras que el embarazo complicaba (Frers, 1919). Aunque entre las del primer tipo (se mencionaron anemia perniciosa grávida, vómitos incorregibles y albuminurias) no todas admitían las mismas posibilidades de un tratamiento exitoso, se realizara o no un aborto, los desarrollos teóricos reconocieron un protocolo ajustado de intervención, más allá del cual habría poca posibilidad de maniobra (Frers, 1919). Otra complejidad se encontró alrededor de las denominadas enfermedades complicadas con el embarazo. En 1919, aparecieron en esa enumeración, además de la diabetes, las cardiopatías graves, el cáncer y la tuberculosis.

En relación con estas dos últimas patologías, llamaron la atención los cambios en las orientaciones médicas, en una época en que sobrevenían persistentes incertidumbres para tratar y curar dichas afecciones, aun en casos en que las mujeres se encontraran internadas para su atención. La tisis y el cáncer constituyen dos patologías paradigmáticas de la agenda de viejas y nuevas enfermedades, característica de una época de transición en la Argentina de las primeras décadas del siglo XX (Armus y Belmartino, 2001). Si bien las afecciones "modernas" como el cáncer comenzaban a posicionarse, hasta 1928 la tuberculosis figuraba como segunda causa de muerte (Armus, 2007, p. 24). En otros términos, durante la etapa en estudio, mientras la incidencia de la tisis aún era relevante, el cáncer avanzaba lentamente experimentando un aumento en su importancia relativa.

Claro que, frente a este panorama de morbimortalidad, los profesionales contaban con algunas notables terapias específicas. En relación con el tratamiento de la tuberculosis, se "listaban [además de] una notable variedad de remedios, de dudosa efectividad algunos, inocuos o incluso perjudiciales otros... las rutinas propias de la cura de reposo, presentes durante todo el período [y] a partir de los años veinte, las intervenciones quirúrgicas, en particular el neumotórax" (Armus, 2007, p. 301). Para el cáncer, en este caso con epicentro en el cuello y cuerpo del útero, imperaba un ambiente médico atravesado por intereses y visiones contrapuestas. Entre los ginecólogos dedicados al diagnóstico y el tratamiento oncológico, gravitó especialmente la división entre quienes defendían la capacidad curativa de la cirugía y los que concebían que la única intervención efectiva descansaba en la aplicación exclusiva de métodos radioterápicos (Rodríguez, 2016). Ahora bien, más allá del relativo avance del conocimiento y la tecnología médicas en estos tiempos, a comienzos del siglo XX las incertidumbres biomédicas continuaban imperando frente a algunas enfermedades que dominaban la morbilidad y mortalidad (Armus, 2016b). La tisis, "hasta la llegada de los antibióticos fue una enfermedad maldita y ante ella una infinidad de terapias sólo mostraron impotencia" (Armus, 2016b, p. 49). En el caso del cáncer, las terapias efectivas tardaron aún más en conocerse.

Si este panorama era complejo para cualquier enfermo, la situación de las pacientes embarazadas las colocaba frente a una situación de mayor vulnerabilidad. En relación con estos casos clínicos, muchas de las argumentaciones señalaron la existencia de brechas entre unos discursos orientados a señalar la utilidad de interrumpir el embarazo cuando corría riesgo la salud o vida de la gestante y, una serie de barreras de pretendido carácter científico, traducidas en obstáculos a la posibilidad de indicar y practicar un aborto terapéutico. Mientras se reconocía la incuestionable legitimidad del aborto terapéutico, se reprodujeron 
constantemente precisiones que definían mayores posibilidades de expectación, interponiendo alternativas terapéuticas a la interrupción del embarazo. Para la tuberculosis, en 1919, las indicaciones fueron desde las críticas de Frers a aquellos profesionales que se oponían el aborto, basados en suponer "...la incurabilidad de la tuberculosis en cualquier período", hasta, fundamentar por la misma razón, que, en algunos casos el aborto no era aconsejable puesto que las lesiones graves y extensas terminan desapareciendo con el mismo tratamiento" (p. 445). Algunos pocos años después, el andamiaje de proposiciones y orientaciones pareció aún menos proclive a reconocer el potencial beneficio que este recurso podía traer a enfermas embarazadas. El mismo año en que el aborto terapéutico fue despenalizado, en 1922, un médico del Servicio de Ginecología del Hospital Rawson de Buenos Aires, se refirió a la tisis como una enfermedad en la que "los peligros de muerte para la madre eran contingencias del futuro". En el mismo escrito, se excluyó al cáncer de la lista de patologías que habilitarían el aborto por razones terapéuticas (Elicegui, 1922, p. 54 y 57).

Durante estos años en que prevalecía la casi inexistencia de derechos para las mujeres, la autoridad de la medicina fue un caldo de cultivo para el ejercicio de un poder-saber médico decididamente androcéntrico. En este entramado, se articularon factores de distinto cuño. No faltaron los cuestionamientos éticos de elegir entre "sacrificar una vida por otra"(Frers 1919, p. 450). Ahora bien, estas nociones se extendieron más allá de las esferas subjetivas, cristalizándose en un conjunto de indicaciones, pretendidamente objetivas, que colocaron en primer lugar la función de la maternidad, a través de imaginarios identificados con las nociones de vivir para y a través de su cría. Para las mujeres con riesgo de salud y vida, esos criterios profesionales eran muy restrictivos. Por un lado, estaba la paciente que por encontrarse en una situación mórbida de gravedad debía renunciar a cualquier intento de restablecerse, sacrificando su vida por la que venía a nacer; para ella, no era estimable de indicación de aborto médico. Por otro, se encontraba la candidata preferida al aborto terapéutico, la madre que no debía morir porque tenían una prole ya existente (Rodríguez, 2018). Sin ambages, se indicó como una orientación para la decisión de los profesionales que averiguaran "si la enferma tiene hijos, porque en este caso el derecho de ellos de conservar a la madre debía prevalecer sobre los derechos de otro de vida supuesta e insegura, capaz de ocasionar la muerte de la madre" (Elicegui, 1922, p. 57 en Rodríguez, 2018, p.200). Es que, a diferencia de las cardiopatías graves o las enfermedades ovulares, en las que se podía esperar la cura mediante el aborto, a las tísicas embarazadas con lesiones muy avanzadas, se las asumía más o menos cerca de la muerte, por tanto, no se justificaba alargarles la vida, sacrificando a su hijo (Elicegui, 1922).

La reforma penal de 1921 no tuvo incidencia directa en la manera en que los médicos trataban la posibilidad o no de anteponer un aborto médico. Con el transcurrir de los años, fue cada vez más visible que las decisiones de los especialistas se inclinaban a preservar la vida de la madre y del hijo, aunque ello, en los hechos, pusiera en riesgo la salud y la vida de la mujer. Concretamente, en el tratamiento de las tuberculosas embarazadas imperaron durante la década de 1920 terapias alternativas al aborto, viabilizadas en los tratamientos con neumotórax o la expectación terapéutica (que simplemente consistía en esperar la evolución de la enfermedad). Estas opciones médicas no habrían formado parte de la "historia oficial" narrada por parte de la medicina actual, la que reconoce que recién abrazó la posición abstencionista para las tuberculosas embarazadas a mediados del siglo XX (Hiriart, Varas y Jiménez, 2006 citado en Rodríguez, 2018).

A pesar que los casos de abortos médicos son escasos en las fuentes, la ejecución de este tipo de intervenciones fueron opciones concretas rastreables empíricamente. El aborto terapéutico practicado en 1926 por los doctores José Bello y Alberto Peralta Ramos a una paciente tuberculosa soltera de 24 años, con cinco meses de embarazo, facilitó comprender cómo los fenómenos socioculturales atravesaron las categorías médicas, definiendo las intervenciones sobre el cuerpo y la experiencia vital de las mujeres enfermas y embarazadas (Rodríguez, 2018). Resultó significativo detenerse a considerar las razones del registro de este caso clínico, que apareció como una publicación de actas de una Sesión de la Sociedad de Obstetricia y Ginecología de Buenos Aires. Allí, Peralta Ramos y Bello justificaron ante sus colegas porqué habían realizado 
un aborto terapéutico utilizando una técnica abdominal, que usualmente se interponía cuando se buscaba completar esa intervención con una esterilización, práctica que no habían procurado realizar estos doctores.

Al considerar este caso, no pasa desapercibido que Alberto Peralta Ramos, profesor de Clínica Obstétrica y jefe de Maternidad del hospital Rivadavia, haya sido reconocido por la historiografía como responsable de varios abortos terapéuticos seguidos de esterilización definitiva, supuestamente fundados en motivos eugenésicos (Eraso, 2007). En efecto, este médico era un reconocido defensor de la necesidad de "evitar el nacimiento de seres enfermos o débiles, con graves defectos o enfermedades hereditarias que imposibiliten la vida individual con capacidades positivas para la sociedad o que acarreen consigo mismo el peligro de contraer o de propagar las enfermedades sobre los que lo rodean" (Nari, 2005, p. 50 en Rodríguez, 2009, p. 80). No obstante, los registros de aquella sesión no indicaron que se pusieran en tensión presuntas tramas de eugenesia “positiva o negativa”. Más bien, las explicaciones de Bello y Peralta Ramos evidenciaron micro prácticas propias de un poder médico disciplinador, que aunó clivajes de violencia explícita e implícita, propias de un conocimiento y una praxis científica con nítidos sesgos androcéntricos.

Concretamente, en la exposición, que llamativamente titularon "Pequeña cesárea en embarazo complicado por tuberculosis e himen intacto", los especialistas compartieron dos momentos clave en sus decisiones, ambos definidos por el interjuego entre concepciones biomédicas y valores sobre el funcionamiento esperable de la sociedad y las relaciones de género. En el primero, recordaron el interrogatorio inicial que le realizaron a la paciente tísica, lo que les habría permitido entender que había conservado el "himen intacto", porque su embarazo fue el resultado de la fricción del pene del novio entre sus muslos. En segundo lugar, las explicaciones de estos médicos giraron alrededor de justificar haber recurrido a la técnica conocida como "pequeña cesárea" -denominación que recibía el método abortivo por vía abdominal-, sosteniendo que, "si hubieran tenido la completa seguridad de que el novio iba a cumplir su compromiso tal vez hubieran seguido la vía baja a pesar que esta puede resultar engorrosa, larga y sangrante tratándose de un feto de varios meses” (Peralta Ramos y Bello, 1926, p. 297 en Rodríguez, 2018, p. 209). De acuerdo con los análisis de Lois (2018, p. 158), se podía conjeturar que en el caso de esa paciente tuberculosa embarazada su condición de soltera le habría dificultado "ser la interlocutora" de los médicos, los que, preocupados por el deshonor de la joven habrían entendido conveniente conservar su himen intacto para la mejor consecución de un futuro matrimonio.

No parece que estos profesionales hubieran tenido que recurrir a algún tipo de estratagema discursiva para conseguir una gran autonomía de acción frente al tratamiento a seguir. En esta etapa, en que la medicina aún no se guiaba por regulaciones, sistemas de convenciones y procedimientos propios de la denominada medicina basada en evidencias, el camino terapéutico más conveniente para tratar a las enfermas embarazadas se decidía según cada caso clínico, es decir para cada mujer en particular. Esta matriz permitió notables discrecionalidades. Por su parte, el artículo 86 no acordó ningún tipo de regla en este sentido, estableciendo vagamente que, para admitir legalmente este tipo de aborto, no debía existir ninguna otra alternativa para salvar la vida o salvaguardar la salud de la mujer (Maffia, 2006).

Para principios de 1920 el tratamiento de gestantes con cáncer en su aparato genital, apareció sujeto a la misma lógica en la autonomía de criterio profesional que se ha verificado para la tisis. Mejor dicho, en aquellos casos esa discrecionalidad eminentemente masculinizada, parecía haber estado aún más extendida. El cáncer para 1922 ya no formaba parte del cuadro de enfermedades en las que se aconsejaba un eventual aborto. Por otro lado, a pesar de la mayor incidencia que venía cobrando la enfermedad entre las causas de muerte, fue exiguo el lugar que ocupó entre los especialistas su asociación con el estado de gestación, lo que de acuerdo al Dr. Carlos Castaño (1918) podría explicarse en que era prácticamente excepcional encontrar embarazos en mujeres con neoplasias en el cuerpo y cuello de su útero. Las contribuciones de Castaño resultaron especialmente significativas, mostrando cómo los médicos católicos obviaron argumentos religiosos, cuando apenas unos años más tarde mantuvieron un discurso orgánico con la Iglesia Católica, abiertamente contrario a la despenalización del aborto por cualquier causa (Rodríguez, 2009). A partir de 
1929 Castaño se incorporó como parte del grupo de 8 médicos que dirigió el Consorcio de Médicos Católicos de Buenos Aires (Rodríguez, 2009, p. 69-70)

Según este ginecólogo, el panorama de literatura médica nacional era bien pobre sobre el tema, lo que podía resumir en "la tesis del Dr. Collillas presentada en 1908 con 6 casos de embarazo y cáncer tratados en el Hospital Rivadavia y en la exposición del Dr. Gabastou en la Asociación Nacional de Parteras en 1911, donde presentó el único caso ocurrido en 4 años en la Clínica Obstétrica del Hospital San Roque" (Castaño, 1920, p. 162). Por su parte, la performance de Castaño no había sido muy distinta a la de sus colegas. En 10 años atendiendo en el Servicio de Ginecología del Hospital Nacional de Clínicas, "donde desfilan anualmente alrededor de 3000 enfermas entre sala y consultorio externo solo se han presentado dos casos" (1920, p.161).

Estos pocos casos registrados no impidieron que algunos hospitales se convirtieran en espacios para que los ginecólogos hicieran uso de las terapias disponibles en la época para tratar a las cancerosas embarazadas. Esta agenda fue muy prolífica en la discusión y experimentación clínica de nuevos criterios y tecnologías para tratar el cáncer, originadas en el ámbito científico internacional. Castaño se visibilizó como el más firme defensor de la radioterapia, dando a conocer una suerte de diálogo particular entre sus experiencias como ginecólogo al pie de la cama y como un académico atento a las transformaciones científicas difundidas especialmente desde algunos centros médicos europeos.

La decisión de indicar un aborto médico en mayo de 1914, en el caso de una mujer de 33 años con cáncer de cuello de útero, se presentó como parte de una etapa cerrada en su vida profesional. En 1917, la internación de una nueva paciente embarazada de 5 meses, también afectada de un cáncer de cuello, aún más virulento que el de la paciente anterior, fue la oportunidad de hacer suyo el criterio sobre que el "radio y sus aplicaciones en el cáncer [habían] venido a revolucionar la medicina y especialmente la Ginecología, considerándose hoy como el único tratamiento verdaderamente eficaz" (Castaño, 1920, p. 164 y 168). En efecto, para 1920 esta mujer completaba una sobrevida de casi 3 años, en un caso que se había comunicado en la Sociedad de Ginecología y Obstetricia de Buenos Aires en el año 1918 como la primera experiencia en la historia médica nacional en que se trató a una gestante con cáncer genital aplicando exclusivamente radio, lo que generó las condiciones clínicas para que el embarazo llegara a su término, “(...) extrayendo mediante cesárea un niño que vive y es sano" (Castaño, 1918, p. 258-259).

Para los primeros tiempos del siglo XX, la curieterapia formaba parte de un proceso de circulación de tecnologías que ocupaba un lugar destacado en la agenda de la ginecología local, sobre todo entre los especialistas, que venía procurando monopolizar el estudio y el tratamiento de estas neoplasias femeninas. No obstante, la adopción de estas nuevas técnicas generó entre los ginecólogos locales acalorados enfrentamientos, rastreados desde los primeros números de la Revista de Argentina de Obstetricia y Ginecología. Las incertidumbres en ese sentido eran múltiples y muy fundamentadas: la dosis ideal, la distancia óptima de irradiación para generar algún efecto sin dañar a la paciente, los filtrajes y los pocos estudios de largo plazo que permitían respaldar resultados curativos por parte del radio y los rayos X, ya que en ninguno se había mostrado una supervivencia en las enfermas que llegara a los 5 años, frente a extendida estadística que ostentaba la amplia histerectomía (Rodríguez, 2016).

Claro que, salvo en los enfrentamientos académicos y en las experiencias clínicas publicadas por destacados especialistas españoles, la fuerte tradición quirúrgica de la ginecología argentina propiciaba que la cirugía y las tecnologías radioterápicas no fueron alternativas excluyentes en los tratamientos realizados en varios de los hospitales más populosos de la ciudad de Buenos Aires. El radio se incorporó en el tratamiento de manera conjunta con la cirugía, especialmente cuando los cánceres de cuello de útero, los más virulentos en su malignidad y evolución, se consideraban dentro de los límites de la operatividad (Rodríguez, 2016).

En este contexto, los planteos del Dr. Castaño, por entonces jefe de Clínica Ginecológica de la Facultad de Medicina habilitarían distintas interpretaciones. Por un lado, habrían representado - en contra de quienes defendían el potencial curativo de la histerectomía - el despliegue de una actitud pionera y permeable frente a la trama circulatoria de saberes y tecnologías en el ámbito de la especialidad. Él secundó de cerca 
la ruptura que en la ginecología madrileña posibilitó el empeño del Dr. Sebastián Recasens, quien desde 1915 difundió los resultados de sus tratamientos con radioterapia sobre casos de cáncer de cuello de útero inoperables (Corbacho, 2008, p. 108 en Rodríguez, 2016, p. 79). Por otro lado, esta emergencia y resignificación de saberes y tecnologías, también involucraba dinámicas que ponían en funcionamiento creencias y convicciones, que se podrían denominar extracientíficas, aunque fueran constitutivas de la formación de criterios y la toma de decisiones terapéuticas.

Es que, si en un principio el cáncer había salido de la lista de patologías susceptibles de aborto porque la interrupción del embarazado no constituía una terapéutica en sí misma, sino que era la consecuencia esperable de los abordajes quirúrgicos que venían siendo dominantes, ahora la aplicación exclusiva del radio desplazaba la intervención quirúrgica, dándole centralidad a una intervención que creaba las condiciones para conservar la gestación o, al menos, no implicada un altísimo riesgo de abortar. Estos empeños reforzaban el poder y los valores socioculturales dominantes entre algunos médicos, influyendo en sus elecciones de aquellos tratamientos que permitían la consecución del producto de la gestación en curso, aunque, de acuerdo a los conocimientos de época, se pusiera en riesgo la salud y la vida de las mujeres.

En distintas ocasiones se puede identificar este recorrido en las posturas de Castaño. Por una parte, aunque, como apuntó Frers (1919) y reafirmó Castaño (1920, p. 162), se consideraba que "el embarazo acelera[ba] la marcha del cáncer de cuello", se minimizó el peso que tendrían las escasas estadísticas locales y extranjeras que apoyaban la capacidad de curar y las evidencias de sobrevida que se manejaban alrededor de la aplicación exclusiva del radio. Por otra, se soslayaban estas discusiones, enfatizando que el interés radicaba en "conocer la conducta a seguir en el tratamiento". En ese sentido, el radio permitiría la curación clínica de la madre y salvar la vida del hijo, cumpliendo así “el ideal más completo" (1920, p. 163). Este logro, según se reafirmó, superaba las posibilidades demostradas por la histerectomía amplia total, cuyos resultados estadísticos exitosos habían hecho que, hasta al mismo Pinard, dejara de “... pensar en la conservación sistemática del feto, para ir, sin pérdida de tiempo a salvar a la madre” (1920, p. 161-164). Este tipo de concepciones se trasladaron a las experiencias clínicas, respaldando la decisión de aplicar sólo radio en el caso de 1917, en su interés por conservar al niño de una paciente que transitaba "su primer embarazo, en un matrimonio que sólo llevaba un año de casados"(Castaño, 1918, p. 259).

Eran constantes las críticas que muchos profesionales esgrimían contra la vaguedad con que los defensores del radio utilizaban el término "curación clínica", así como sus acusaciones sobre cómo aquellas escuetas estadísticas llamativamente presentaban un bajísimo porcentaje de pacientes presentados en condiciones de operabilidad (Villar, 1919 citado en Rodríguez, 2016). De todas maneras, no pasa desapercibido que, ante la inexistencia de técnicas de diagnóstico precoz efectivas, las que no surgieron antes de la segunda posguerra (Eraso, 2014), era usual que los ginecólogos trataran cánceres muy avanzados. En el caso de las neoplasias asociadas a situaciones de embarazo, fue una constante que las mujeres concurrieran por primera vez a la consulta con una gravidez muy avanzada. Seguramente, perduraban las usanzas propias de fines del siglo XIX, cuando “(...) el embarazo sólo podía ser identificado por la mujer (generalmente cuando el feto comenzaba a moverse, alrededor del quinto mes de gestación) (...)" (Arosteguy, 2018, p. 143). Aunque ya se realizaban algunas pruebas con orina desde siglos atrás, hasta la década del treinta, y sobre todo del cuarenta en América Latina, no se estableció la relación de la hormona HCG (Gonadotropina Coriónica Humana) con el embarazo (Lois, 2018). Asimismo, aunque en la década del treinta, más del 60\% de los partos ya se realiza en instituciones médicas (Nari, 2004 citado en Lois, 2018) los controles prenatales habrían estado muy pocos extendidos.

Más allá del caso de aquella paciente de 1917, la que había llegado a la consulta con 5 meses de embarazo y con un cáncer muy avanzado y virulento, situación que le habría valido estar fuera de los límites de la cirugía, Castaño también apoyaba la aplicación únicamente del radio cuando el caso "complicado de embarazo" fuera operable (1920, p. 166-167). En estos años, se advertía que el panorama de criterios y prácticas en las intervenciones en los cánceres operables era heterogéneo. Era usual encontrar que unos médicos “... hacen 
radiaciones exclusivamente; otros operan y luego hacen radiaciones profilácticas" (Harguindeguy, 1919, p. 111 citado en Rodríguez, 2016, p. 71).

La extensión en la atención hospitalaria durante la década de 1920, no introdujo transformaciones radicales en las dinámicas repasadas. Las incertidumbres y la autonomía de criterios parecían seguir enmarcando los tratamientos médicos que recibían las gestantes con cáncer. En 1925 el Dr. Pedro Ferrazini (1925) presentó en la Sesión de la Sociedad de Obstetricia y Ginecología de Buenos Aires un caso clínico indicador de estas persistencias. En esta ocasión, relató el caso de una mujer a la que, luego de tener un parto normal, se le realizó una biopsia "con la cual se hizo el diagnóstico de epitelioma maligno" (1925, p. 227). No todos los ginecólogos sostenían las mismas interpretaciones ante manifestaciones mórbidas similares. De hecho, este profesional admitió que la histerectomía en casos de cáncer de cuello de útero era una posibilidad aceptada, incluso hasta el sexto mes de gestación. Claro que esta postura no tuvo ninguna relevancia concreta en ese caso clínico, ya que el diagnóstico se había hecho con un embarazo muy avanzado y el progreso del carcinoma se consideraba fatal (1925, p. 227). Difícilmente se entienda la suerte que corrió esta mujer mientras esperaba su muerte sino recapitulamos algunas de las proposiciones que publicó Frers muchos años antes, cuando señaló que frente al cáncer inoperable habría "que abstenerse de toda intervención paliativa que no hará sino comprometer la vida ya amenazada del feto sin beneficiar suficientemente a la madre que está irremisiblemente condenada" (1919, p. 448).

\section{Consideraciones Finales}

Las argumentaciones en circulación desde los primeros números de la Revista Argentina de Obstetricia y Ginecología reafirman que, aunque estos especialistas locales sostuvieron cierta apertura frente a la interposición del aborto cuando la salud o la vida de la mujer/madre corría riesgo, las posturas predominantes remitieron a concepciones y actitudes restrictivas. Aunque eventualmente se practicaron abortos por razones médicas, las indicaciones que orientaban el tratamiento de la tísicas y cancerosas abrazaron el criterio de aconsejar terapias alternativas al aborto.

Como se ha procurado mostrar, insistiendo en una lectura en clave de género, la preeminencia de la matriz transformista identificada con la "eugenesia positiva" respondió a dinámicas que, bajo el poder médico, moldearon la intersección entre biología y política. En ese sentido, pierde consistencia elaborar una especie de jerarquía analítica entre argumentaciones discursivas y acciones, como si las primeras carecieran de un estatus performativo. Tal como afirmó Maffia (2007, p. 70) no puede negarse que las ciencias biomédicas con sus teorías "sólidamente establecidas .... influyen en la vida cotidiana de las mujeres tanto como en la determinación de su lugar social, político y jurídico".

Desde antes de la despenalización de aborto, la elite médica comenzó a posicionarse frente a ese recurso a partir de criterios atravesados por nociones extracientíficas. Las actitudes de cautela legal dejaron en evidencia cómo los especialistas definieron al aborto como un asunto de entera incumbencia profesional. Sin duda, ello se explica en un marco de ausencias de derechos para las mujeres, de acuerdo con las tracciones patriarcales que definieron los procesos de medicalización del cuerpo femenino. La insistencia en una nítida distinción entre lo religioso y lo secular, si bien refería a una real distinción entre las normas religiosas y el derecho moderno, también implicó ciertas continuidades en lo que respecta al proyecto de poder de diseño patriarcal. La sanción de 1922 no puso fin a esta situación, sino que inició un largo camino de vacíos, propios de un marco legal sin guías de procedimientos ni garantías.

En la medicina, este proceso de autonomía se apuntaló en la preeminencia del criterio clínico "caso a caso". Ligado a este poder médico, las decisiones sobre los caminos terapéuticos más adecuados priorizaron la agenda del desarrollo científico de la época, pero al hacerlo se soslayaron las incertidumbres en cuanto a las posibilidades de restablecer la salud o salvar la vida de las pacientes. Los avances tecnológicos se imbricaron 
con las normativas morales, poniendo en evidencia la contracara de los postulados principios de objetividad, neutralidad y universalidad de la ciencia (Maffia, 2007).

Por esta vía los profesionales colocaron en primer término las terapias alternativas al aborto,incluso con el riesgo que ello implicaba para la mujer enferma. A pesar de la inexistencia de protocolos, durante estos años el criterio clínico estuvo orientado por indicaciones especializadas, presentadas como objetivas. Los contenidos de estos trabajos científicos se trasladaron a las decisiones y prácticas terapéuticas concretas, operando como barreras persistentes al acceso al aborto terapéutico. Allí, confluyeron criterios especializados, representaciones y mandatos patriarcales que, en situaciones como el embarazo, pusieron en evidencia las normativas masculinizadas sobre el ejercicio de la sexualidad y los comportamientos sancionables en el uso del cuerpo. El manejo de terapias alternativas al aborto, no sólo propuso como prioridad conservar la gestación y posibilitar la maternidad. En los hechos, se accionaban decisiones y prácticas médicas que articulaban concepciones, criterios y tecnologías que buscaron ejercer control sobre la mujer. A partir de ese universo se disponía qué vidas y qué familias merecían perdurar.

Huelga decir, ello no cambió luego de la reforma penal de 1921. De hecho, el Código Penal contribuyó a reproducir esas mismas estructuras desde las que se establecieron controles sociales sobre las mujeres, su sexualidad y su cuerpo (Zulita Fellini y Sansone, 1999). Efectivamente, en este contexto, donde la mujer apenas si gozaba de ciertos atributos de ciudadanía, los obstáculos interpuestos para al acceso a este tipo de aborto legal, se sumaron como un renglón más de las perdurables relaciones de dominación de género. Claro que, estos valores y normativas androcéntricas, adquirieron ribetes fatales en las experiencias de estas mujeres enfermas. Ellas, frente a un saber/poder que define cuándo hay riesgo de salud o vida y, por supuesto, cuándo y porqué es aconsejable o no el aborto, tuvieron menos autonomía para decidir que otras, presumiblemente sanas y en circunstancias que reclamaban un aborto. De todas formas, en cualquiera de estos casos, el Derecho y la práctica médica operarían condicionados por una violenta desigualdad de género.

\section{ReFERENCIAS}

Armus, D. (2007). La ciudad impura: salud, cultura y tuberculosis en Buenos Aires, 1870-1950. Buenos Aires: Edhasa.

Armus, D. y Belmartino, S. (2001). Enfermedades, médicos y cultura higiénica. En A. Cattaruzza (dir.), Crisis económica, avance del Estado e incertidumbre política, Tomo VII., Nueva Historia Argentina (pp. 283-330). Buenos Aires: Sudamericana.

Armus, D. (2016a). Eugenesia en Buenos Aires: discursos, prácticas, historiografía. História, Ciencias, SaúdeManguinhos, 23 (Supl.), 149-169.

Armus, D. (2016b). Medicina casera, remedios y curanderos en los inicios de la medicalización de la ciudad moderna. Buenos Aires, 1870-1940, Tempos Históricos, 20, 47-80.

Arosteguy, J. (2018). Constitución y aborto. Notas sobre la inconstitucionalidad de la penalización. En Busdygan, D. (Coord.). Aborto. Aspectos normativos, jurídicos y discursivos (pp. 141-156). Buenos Aires: Biblos.

Beruti, J. (1919). Aborto terapéutico. Sesiones de la Sociedad de Ginecología y Obstetricia de Buenos Aires, 16 y 17 de octubre de 1919, Revista Argentina de Obstetricia y Ginecologia, III(6), 480-482.

Biernat, C. y Ramacciotti K. (2013): Crecer y multiplicarse: La politica sanitaria materno infantil, Argentina 1900-1960. Buenos Aires: Biblos.

Calandria M. S. y Ledesma Prietto N. F. (2018). Abortos e infanticidios: tensiones y debates en la legislación penal moderna (1886-1968). Avances del Cesor, XV(19), 101-128.

Castaño, C. A. (1918). Cáncer y embarazo.Sesiones de Sociedad de Obstetricia y Ginecología de Buenos Aires, 19 de junio de 1918. Revista Argentina de Obstetricia y Ginecologia, II(1), 258- 259.

Castaño, C. A. (1920). Cáncer y embarazo. Revista Argentina de Obstetricia y Ginecología, IV(1), 162-168. 
Centro de Estudios Legales y Sociales (CELS) (2016). Situación del aborto en argentina. evaluación sobre el cumplimiento de la convención para la eliminación de todas las formas de discriminación contra las mujeres $(C E D A W)$. Buenos Aires: Informe.

Cepeda, A. (2017). Los abortos no punibles. Argumentos médico-jurídicos y bioéticos en la Argentina de fines del siglo XX.Descentrada, 1(2), e019. Recuperado de http://www.descentrada.fahce.unlp.edu.ar/article/view/DESe019

Elicegui, D. (1922). Algunas consideraciones sobre el aborto terapéutico, Revista Argentina de Obstetriciay Ginecología, $V I(1), 54-62$.

Eraso, Y. (2007). Biotypology, endocrinology, and sterilization: the practice of eugenics in the treatment of argentinian women during the 1930s. Bulletin of the History of Medicine, 81(4), 793-822.

Eraso Y. (2014). Controlling female cancer in Argentina. Divergentinitiatives and the road to fragmentation. Dynamis, 34(1), 73-99.

Fellini, Z. y Sansone V. (1999). La mujer en el derecho penal argentino. Anuario de Derecho Penal, 1999-2000,1-29.

Ferrazini, P. (1925). Cáncer del Cuello de Útero y embarazo. Sesiones de la Sociedad de Ginecología y Obstetricia de Buenos Aires, 14 de mayo de 1925. Revista Argentina de Obstetricia y Ginecología, IX(1), 227

Frers, A. (1919). Aborto terapéutico, Revista Argentina de Obstetricia y Ginecología, III(5), 430-451.

Giordano, V. (2013). La sanción de la capacidad civil plena de la mujer en los países del Cono Sur, 1945- 1990. Una propuesta de análisis del fenómeno legal. Latin American Research Review, 48(3), 25-43.

González, Juan B. (1934) El aborto terapéutico con motivo de un proyecto de ley de la provincia de Córdoba. $L a$ Semana Médica XLI. 2, 177-178.

Lois, I. P. (2018). Medicina y Maternidad: Pariry nacer en la Buenos Aires de inicios del siglo XX (Tesis doctoral inédita). Universidad Nacional de San Martín, San Martín.

Nari, M. (2004). Politicas de maternidad y maternalismo politico: Buenos Aires (1890-1940). Buenos Aires: Biblios.

Maffia, D. (2006). Aborto no punible: ¿Qué dice la ley argentina?”. En S. Checa (Comp), Realidades y coyunturas del aborto. Entre el derecho y la necesidad (pp.1-6). Buenos Aires: Paidós.

Maffia, D. (2007). Epistemología feminista: La subversión semiótica de las mujeres en la ciencia. Revista Venezolana de Estudios de la Mujer, 12(28), 63-98.

Queirolo, G. (2013). Género y sexualidad en tiempos de males venéreos(Buenos Aires, 1920-1940. Revista Nomadias, 17, 67-87.

Rodríguez, A. M. T. (2009). Nuestra patria está en peligro: las cunas están vacías. Catolicismo y natalismo en Argentina en los años treinta del siglo XX. Secuencia, 75, 69-95

Rodríguez, M. L. (2016). La terapéutica en el cáncer cervical: Debates de la ginecología argentina en la adopción de la radioterapia entre 1917 y 1919. EäJournal, 8(1), 63-92

Rodríguez, M. L. (2018). Medicina, eugenesia y género: el aborto terapéutico en las mujeres con tuberculosis. Buenos Aires, 1920-1930. Anuario Colombiano de Historia Social y de la Cultura, 45(1), 191-213

Rosenberg, M. (2010). Sobre el aborto no punible. En Congreso de Paises del Mercosur sobre Bioética y Derechos Humanos. Derecho a la salud. Foro por los Derechos Reproductivos Secretaría de Derechos Humanos. Buenos Aires: Ministerio de Justicia de la Nación, 1-16. 